\title{
Dose-dense AC Regimen
}

National Cancer Institute

\section{Source}

National Cancer Institute. Dose-dense AC Regimen. NCI Thesaurus. Code C138035.

A dose-dense chemotherapy reg imen consisting of doxorubicin and cyclophosphamide, used as a neoadjuvant or adjuvant treatment for breast cancer. 